\section{Some mechanisms of working memory may not be evident in the human EEG}

\author{
Emrah Düzel \\ Department of Neurology II, Otto-von-Guericke University, DE 39120 \\ Magdeburg, Germany. emrah.duezel@medizin.uni-magdeburg.de
}

\begin{abstract}
Ruchkin et al. use brain-activity data from healthy subjects to assess the physiological validity of a cognitive working memory model and to propose modifications. The conclusions drawn from this data are interesting and plausible, but they have limitations. Much of what is known about the neural mechanisms of working memory comes from single neuron recordings in animals, and it is currently not fully understood how these translate to scalp recordings of EEG.
\end{abstract}

In this commentary, I outline four types of physiological limitations to what can be concluded from the existing brain-activity data, such as that used by Ruchkin et al.

First, our current knowledge about the neural underpinnings of scalp-recorded EEG signals is not enough to conclude that a neural phenomenon does not exist. Is it legitimate to rule out the existence of specialized short-term memory buffers because their signatures are not evident to us in the EEG phenomena we select? This is especially difficult to judge, as we do not yet have a sufficient understanding of the basic neural mechanisms that underlie cognitive concepts such as "representation" and the "activation" thereof, let alone their reflections in the scalp EEG. Ruchkin et al. suggest that activations of semantic representations, for example, might be deducted from modulations of the N400 component. But it is as yet unclear whether the neurophysiological indices of these activations are DC-shifts. Stimulus-specific persistent neural activity as a neural mechanism underlying working memory was discovered thirty years ago, and it is neural firing that is hypothesized to be sustained by synaptic reverberation (Wang 2001). Oscillations of local field potentials associated with such reverberations might give rise to DC-shifts (Caspers et al. 1987), but they might be more directly visualized as EEG oscillations. The extent to which neural firing itself is visible in the scalp-recorded EEG is probably very limited (Logothetis et al. 2001). Ruchkin et al. acknowledge the importance of neural oscillations, but they limit their use to assessing interareal coupling and prefer DCshifts as an index of intra-areal processing. Why not treat oscillations as an index of intra-areal processing too? It is quite reasonable to assume that certain types of neural oscillations do not covary with slow neural changes, such as DC-shifts, and can reveal neural processes that otherwise remain undetected (Düzel et al. 2003).

Second, one element of physiological working memory models in animals is the robustness of delay activity to distracters (Miller et al. 1996). For example, the Miller et al. study showed persistent stimulus-specific neural firing in inferotemporal cortex as well as prefrontal cortex (PFC), but only the prefrontal activity was robust to distracters in the delay or retention interval. This finding suggests that PFC neurons can maintain stimulus-selective delay activity even when delay activity in inferotemporal regions is disrupted by intervening distracters, which in turn might suggest that stimulus-selective delay activity in PFC does not require delay activity in temporal or posterior brain regions (Goldman-Rakic 1995; Wang 2001). Currently we cannot tell if the DC-shifts recorded by Ruchkin et al. would also show robustness to distracters.

Third, animal studies suggest that prefrontal neurons code information in working memory that is more than a "pointer" to posterior stimulus-specific delay activity. My understanding of how Ruchkin et al. view "pointers" is that these do not store stimulusselective information. Rather, they index where stimulus-selective information is stored. However, there is evidence that prefrontal neurons indeed store stimulus-selective information and that, contrary to what Ruchkin et al. assume, the firing patterns of prefrontal and parietal neurons could be compatible with duplication of information in both regions. A direct comparison of prefrontal and parietal delay activity in nonhuman primates in a spatial working memory task has shown that neurons in both cortical areas exhibit very similar sustained activity during the delay period, with nearly identical spatial tuning (Chafee \& Goldman-Rakic 1998). This finding means that different prefrontal neurons have different spatial selectivity in the delay period and hence can code stimulus-specific information to an extent that is likely to go beyond being a mere pointer to other representations.

Fourth and finally, brain-activity data in healthy subjects cannot identify brain processes that are critical for a given cognitive function. Are the posterior DC-shifts that Ruchkin et al. have related to the maintenance of stimulus-specific information, epiphenomenal, or do they reflect critical processes for working memory? From Ruchkin et al.'s model this question will be difficult to test, because the model suggests that delayed maintenance of stimulus-specific information is accomplished in those brain regions that initially process the task-relevant aspects of the stimulus. A permanent lesion in such brain regions would impair stimulus processing even before any maintenance operation could start. What is necessary to answer this question, are "dynamic" lesions, which cause impairment selectively and transiently during maintenance. One way to achieve such dynamic lesions in humans would be by transcranial magnetic stimulation (TMS) (Düzel et al. 1996) applied during the retention interval over the areas where the posterior DC-shifts occurred in the Ruchkin et al. study. Two recent TMS studies are relevant in this respect. Both of them show that TMS over posterior neocortex disrupts working memory, but only if it is applied early, close in time to stimulus processing, and not later in the retention interval (Harris et al. 2002; Oliveri et al. 2001). One study shows that frontal TMS disrupts working memory only when applied later in the retention interval (Oliveri et al. 2001). These results are compatible with frontal areas acting as working memory stores and posterior areas acting as initial processors rather than regions of activated representations. 